\title{
TONATIUH, UN TRAZADOR DE RAYOS BASADO EN SIMULACIÓN DE MONTE CARLO Y CON LICENCIA GNU GPL
}

\author{
Luis G. Palacín, Amaia Mutuberria, Iñigo Les, Michael Burisch, Ana Bernardos, Marcelino Sánchez \\ CENER (Centro Nacional de Energías Renovables), Departamento de Energía Solar Térmica, \\ C/ Ciudad de la Innovación, 7, 31621, Sarriguren, Navarra. \\ \{lgomez, amutuberria, iles, mburisch, abernardos, msanchez\}@cener.com
}

\section{Resumen}

Tonatiuh es un trazador de rayos, basado en simulación de Monte Carlo. Es especialmente útil para el cálculo de mapas de flujo y eficiencias ópticas de cualquier sistema termosolar, incluyendo estructuras complejas y campos de heliostatos. Pretende estar en la vanguardia de las herramientas de simulación de plantas termosolares. Desde 2006, el desarrollo de Tonatiuh está liderado por el departamento de Energía Solar Térmica del CENER. Tonatiuh trabaja bajo licencia GNU GPL, es de código abierto y su uso es libre. Desde el CENER se anima a la utilización de Tonatiuh, así como a la participación en su desarrollo y extensión, especialmente a las universidades, centros tecnológicos y empresas del sector. El presente documento muestra algunas de las herramientas y aplicaciones incorporadas en Tonatiuh, a través de dos ejemplos.

Palabras clave: Trazador de Rayos, Simulación de Monte Carlo, CSP, Energía Solar Térmica, GNU GPL.

\section{ENERGÍA SOLAR TÉRMICA DE CONCENTRACIÓN}

La necesidad mundial de fomentar las energías limpias y renovables, así como de reducir la dependencia de combustibles fósiles, es una idea ampliamente aceptada [3, 19]. Entre las energías renovables, la energía solar es una de las más prometedoras, debido a su distribución global y su abundancia (se estima que solo el $0.02 \%$ de la radiación incidente en la Tierra sería suficiente para abastecer las necesidades energéticas mundiales). En las centrales térmicas solares de concentración (conocidas por plantas CSP por su acrónimo en inglés), se genera energía eléctrica en los ciclos termodinámicos (típicamente Brayton y Rankine), aprovechando la energía térmica suministrada a un fluido caloportador (aire, agua, sales fundidas...). Esta energía térmica se obtiene concentrando la radiación del Sol mediante diferentes configuraciones de espejos [12]. La mayoría de plantas CSP en funcionamiento están basadas en captadores parabólicos, pero las plantas de torre son cada vez populares, gracias a su mayor eficiencia y menores costes [14]. La operación de las plantas CSP se puede mejorar considerablemente si son combinadas con: 1) sistemas de almacenamiento energético, que permiten mantener la generación de energía eléctrica fuera de las horas solares [10]; y 2) otras fuentes y tecnologías de generación eléctrica [18].

España es líder mundial en la energía solar térmica de concentración, con alrededor del $50 \%$ de la potencia mundial instalada [19]; especialmente gracias al elevado número de plantas CSP de captadores parabólicos que fueron construidas entre 2008 y 2012, debido a las favorables condiciones de la legislación vigente en dicho periodo. Pero este crecimiento se ha visto drásticamente ralentizado por cambios en la reglamentación. Si bien, la posición de líder en el sector, solo se podrá conservar si España realiza la investigación y desarrollo necesarios.

El departamento de Energía Solar Térmica (ST) del Centro Nacional de Energías Renovables (CENER) trata de fomentar el uso de las tecnologías termosolares, así como su penetración en el mercado. Ofrece asistencia y asesoramiento técnico a empresas e instituciones, presta servicios de investigación sobre la energía solar térmica, y colabora activamente en varios comités de normalización y certificación del sector.

El departamento de ST ha desarrollado diferentes herramientas y librerías de simulación de plantas CSP, entre las que destaca el software Tonatiuh. Se trata de una herramienta de simulación para el cálculo de la radiación incidente en sistemas termosolares. Es del tipo de trazadores de rayos, y está basado en simulación de Monte Carlo.

Las ideas principales que han inspirado al desarrollo de Tonatiuh son: 1) desarrollar una herramienta capaz de simular todo tipo de plantas termosolares de concentración; 2) que sea una herramienta intuitiva, fácil de aprender y utilizar; 3) que sea fácil de mantener y mejorar; y 4) que sea uno de los trazadores de rayos más utilizados. 
Tonatiuh es gratuito, y trabaja bajo licencia pública general (GPL) de GNU. Desde el CENER se anima a otros centros de investigación, universidades y empresas del sector, a que lo usen y a que colaboren en su desarrollo.

El presente documento se enfoca en el software Tonatiuh, y está organizado de la siguiente forma. En primer lugar, la sección 2 explica brevemente los tipos de herramientas de simulación de plantas CSP, presentes en el mercado. Mientras que la sección 3 sintetiza las ideas básicas en las que se basa Tonatiuh. A continuación, las secciones 4 y 5 muestran dos ejemplos del uso de dicha herramienta: un captador cilindro parabólico y un campo de heliostatos. Por último, las secciones 6 y 7 resumen las futuras mejoras que se pretender llevar a cabo en Tonatiuh, así como las conclusiones.

\section{HERRAMIENTAS DE SIMULACIÓN DE PLANTAS CSP}

Básicamente, existen dos tipos de herramientas para el cálculo de la radiación incidente en una planta termosolar: 1) herramientas basadas en productos de convolución; y 2) trazadores de rayos.

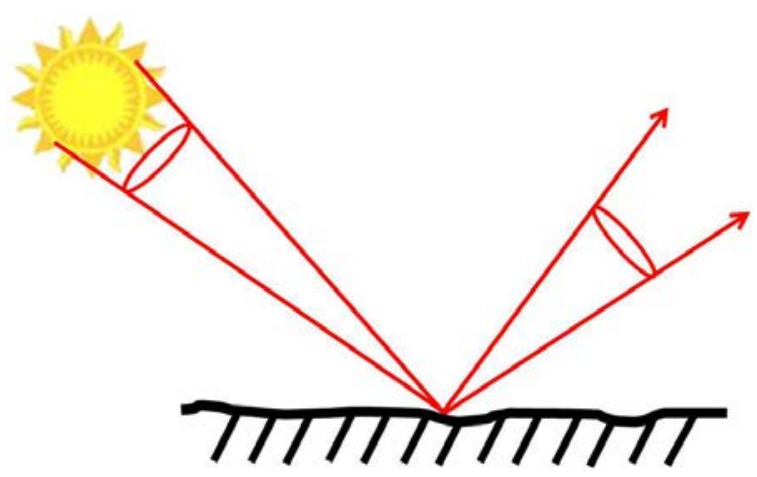

Figura 1: Radiación incidente del Sol y error de la superficie, modelados mediante conos.

En los simuladores basados en productos de convolución, los rayos procedentes del Sol y el error óptico de las superficies, se modelan mediante conos, como trata de representarse en la figura 1. La radiación procedente del Sol, y reflejada en una superficie, se calcula mediante la convolución de estos conos. Por último, para calcular la radiación incidente en el receptor, es necesario calcular la intersección entre la superficie del receptor y el cono correspondiente. Estos simuladores son muy rápidos, pero están limitados a un número pequeño de receptores, formados además, por superficies sencillas. Algunos de los simuladores más populares de este tipo son, por ejemplo, los software DELSOL [8] y HELIOS [2].

Por otro lado, los trazadores de rayos se basan en simulación de Monte Carlo. Calculan trayectorias aleatorias de un número elevado de rayos, que inciden y se reflejan en las distintas superficies. Al contrario que las herramientas basadas en productos de convolución, son mucho más lentos; sin embargo, no están limitados a sistemas sencillos. Entre los trazadores de rayos más conocidos, se pueden citar SolTRACE [24], SOLFAST [20] y Tonatiuh.

Se puede ver una comparación entre los principales simuladores de plantas termosolares en la bibliografía [6, 9, 25]. Si bien Tonatiuh trabaja de manera similar a otros trazadores de rayos, es (en base del conocimiento de los autores) el único de código abierto y bajo licencia GNU GPL. Además, es el más rápido de los trazadores de rayos gratuitos [4].

Tonatiuh está escrito en $\mathrm{C}++$, utilizando la plataforma Eclipse IDE. En su desarrollo, se han utilizado otras librerías y herramientas de código abierto ya existentes. Como por ejemplo, Nokia QT (para la interface gráfica), Coin3D Toolkit (para la visualización en 3D), Marble (para la generación de mapas geográficos) y CPPUnit (para el testeo del código).

Finalmente, Tonatiuh ha sido validado con datos experimentales de la PSA (Planta Solar de Almería, España) [5] y del CNRS-PROMES, en Font Romeu Odeillo (Francia) $[15,16]$. Pero se pretende extender su validación con nuevos estudios.

\section{TONATIUH}

Tonatiuh dispone de una librería gráfica, con diferentes superficies y geometrías tridimensionales sencillas (cilindros, conos, esferas, toroides, prismas, superficies parabólicas, y un largo etcétera). El usuario puede construir geometrías complejas, como una combinación de estos elementos básicos.

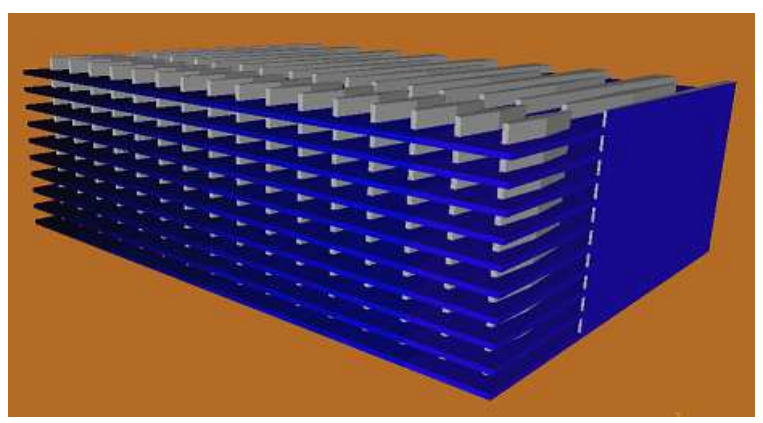

Figura 2: Receptor volumétrico formado por una matriz de placas, modelado con Tonatiuh [22]. 
Tonatiuh está basado en objetos, y permite que aquellas figuras formadas por $n$ elementos idénticos, puedan generarse por un único elemento común y una repetición de punteros, disminuyendo considerablemente las necesidades computacionales. Como ejemplo, la figura 2 muestra el modelo de un receptor volumétrico, formado por varias placas dentadas superpuestas [22]. La radiación solar es concentrada sobre el receptor, y el aire que circula a través de las hendiduras recoge dicho calor.

A continuación, el usuario define las propiedades ópticas de las distintas superficies: principalmente reflectividad, transmisividad y error óptico. Estas propiedades pueden definirse constantes o proporcionales al ángulo de incidencia [11]. Por otro lado, cuando un rayo incide en una superficie y se refleja, el ángulo de reflexión es igual al de incidencia $(\theta)$ más un cierto valor $(\varepsilon)$ proporcional al error óptico. Típicamente, este valor se modela mediante una distribución normal con media cero y desviación estándar fijada por el usuario, como se representa en la figura 3 .

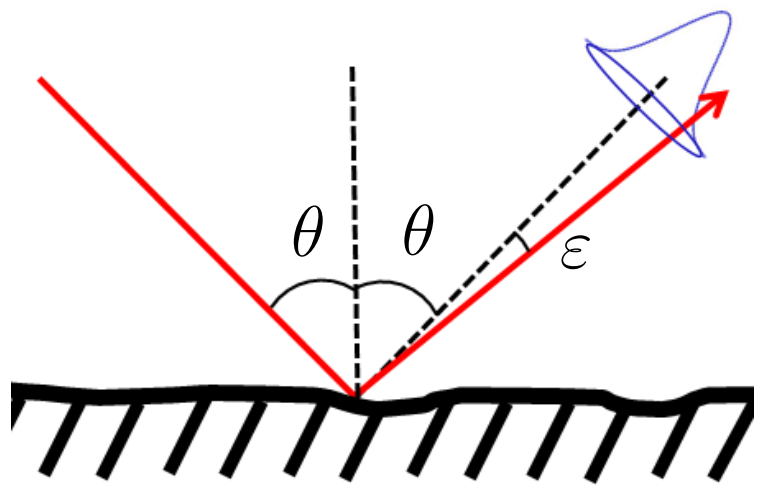

Figura 3: Rayo reflejado en una superficie irregular.

Por último, el usuario define la posición del Sol y las propiedades atmosféricas. Tonatiuh dispone de distintos modelos para la forma del Sol [7] y para la atenuación atmosférica $[1,21,23]$. Además, el usuario puede fijar la irradiancia (conocida por su acrónimo en inglés DNI), y el ratio circunsolar [7].

Una vez definido el sistema, el usuario puede calcular la radiación que le llega a cada una de las superficies, mediante una simulación de Monte Carlo. Tonatiuh calcula caminos estocásticos, para rayos que parten del Sol y llegan a las distintas superficies. Desde la posición del Sol, Tonatiuh lanza un elevado número de rayos hacia el sistema. Cada rayo, impactará en alguna de las superficies que forma la planta, donde se absorberá, reflejará o refractará, en función de las propiedades ópticas de la superficie correspondiente. A continuación, el rayo podrá impactar en otra superficie o salirse del volumen de control. El proceso se repite, hasta que todos los rayos se han absorbido en alguna superficie o han terminado saliendo del volumen de control. Es necesario definir correctamente el número de rayos simulados, para conseguir una buena convergencia de los datos sin sobredimensionar los cálculos. Existen diferentes técnicas para disminuir la varianza de los resultados, agilizando la convergencia. Por ejemplo, a la hora en que Tonatiuh selecciona el origen de los rayos, en la posición del Sol.

Tonatiuh es una aplicación multiplataforma, y puede ser ejecutado en los sistemas operativos más importantes. En particular, se ha probado en los sistemas operativos Windows XP y posteriores, Linux (distribuciones openSUSE 10.x, Ubuntu 12.x y posteriores), y Mac OS X.

La última versión de Tonatiuh, se puede descargar de manera gratuita desde la página:

https://github.com/iat-cener/tonatiuh

\section{CAPTADOR CILINDRO PARABÓLICO}

La figura 4 muestra el modelo gráfico de un captador cilindro parabólico. El fluido caloportador circula por el tubo receptor (amarillo), localizado en el foco de la parábola. El tubo es modular y está recubierto por una cubierta transparente, donde se hace vacío. Los espejos (azul verdoso) están formados por una serie de facetas con forma de cilindro parabólica. Los rayos solares que incidan perpendicularmente a los espejos se reflejarán en la dirección del tubo receptor. El captador gira radialmente siguiendo la posición del Sol, para maximizar el calor incidente.

La eficiencia del captador depende de muchos factores. Entre ellos, destacan los siguientes: 1) El captador solo tiene un eje de giro, y no puede situarse perpendicular a los rayos del Sol. Cuanto mayor sea el ángulo de incidencia, hay una mayor probabilidad de que los rayos se reflejen sin incidir con el tubo receptor. 2) Existen errores en el seguimiento del Sol. 3) El tubo receptor puede no estar exactamente en el foco de la parábola. 4) Parte de los rayos incidentes en los espejos, se absorben. Y parte de los que inciden en la cubierta transparente, no se refractan hacia el tubo reflector. 5) Los errores ópticos y las rugosidades de las superficies hacen que parte de los rayos reflejados no choquen contra el tubo receptor. 6) Parte de los rayos son bloqueados por las conexiones y soportes del captador. Etcétera. Todos estos factores hacen que el cálculo explícito de la radiación incidente en 


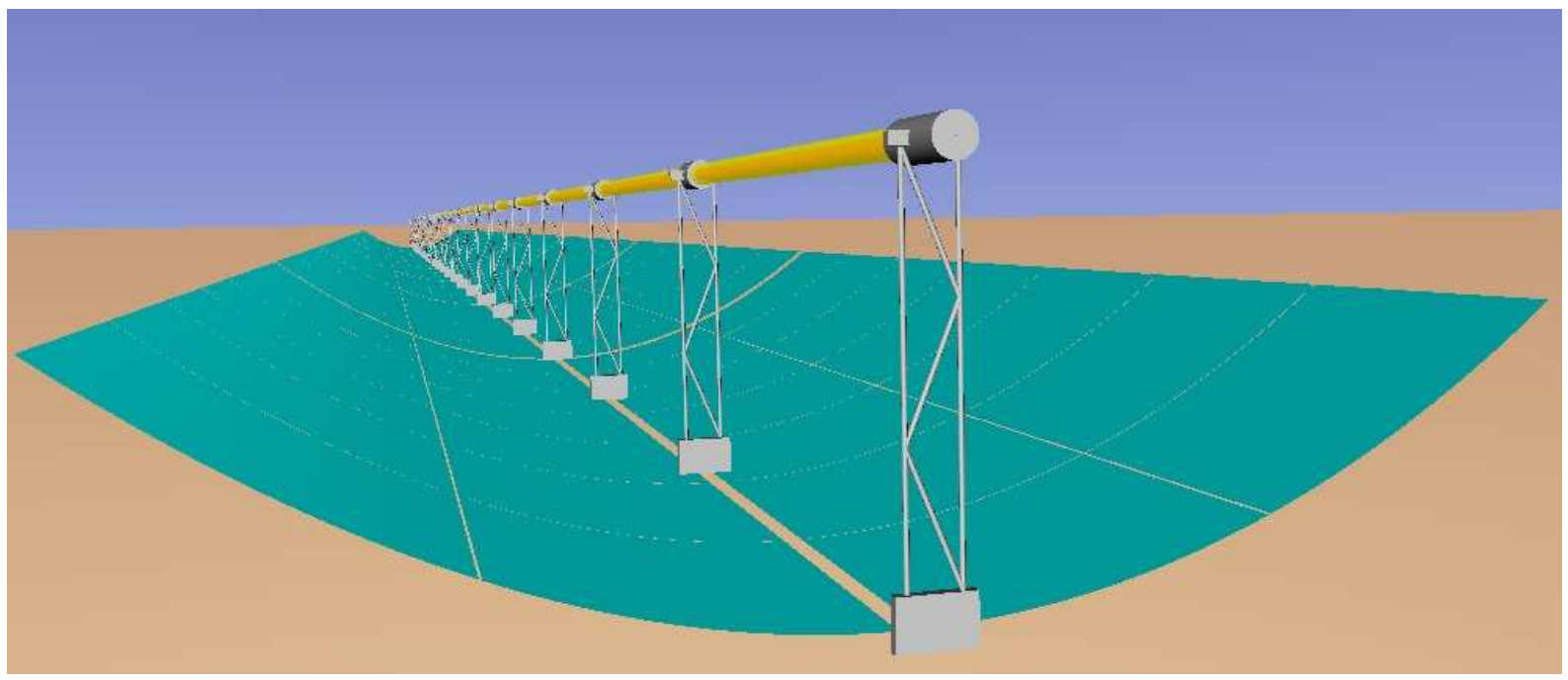

Figura 4: Captador cilindro parabólico, modelado en Tonatiuh.

el receptor sea muy complejo. Pero la naturaleza del problema hace que su cálculo mediante simulación de Monte Carlo sea especialmente adecuado. La figura 5 muestra el trazado de algunos rayos simulados con Tonatiuh.

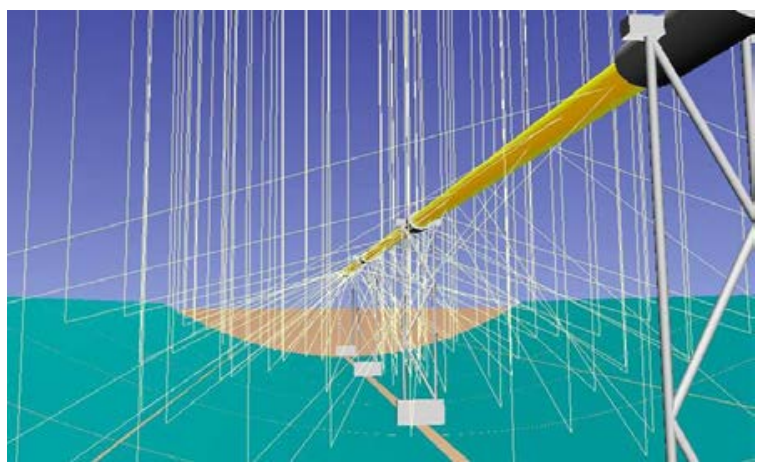

Figura 5: Caminos estocásticos recorridos por los rayos solares, en un captador cilindro parabólico.

Junto al cálculo de la potencia total incidente en el tubo receptor, Tonatiuh permite estimar de manera sencilla otras variables importantes que caracterizan los captadores cilindro parabólicos. Como el modificador de ángulo (conocido por su acrónimo en inglés IAM), y la eficiencia óptica. El IAM se define como el ratio entre la potencia total incidente en el tubo receptor, cuando los rayos inciden en el captador con un cierto ángulo, y la potencia total cuando los rayos inciden perpendicularmente. Mientras que la eficiencia óptica se define como el porcentaje (en tanto por uno), de los rayos que habiendo llegado a los espejos, después de reflejarse, terminan incidiendo en el tubo receptor, respecto del número total de rayos que llegan a los espejos.
Por último, Tonatiuh se puede utilizar para el estudio fotogramétrico de los espejos. La figura 6 muestra varias facetas generadas por combinación de triángulos, obtenidos a partir de las posiciones medidas en la planta, a partir de las irregularidades y deformaciones que puede sufrir el sistema.

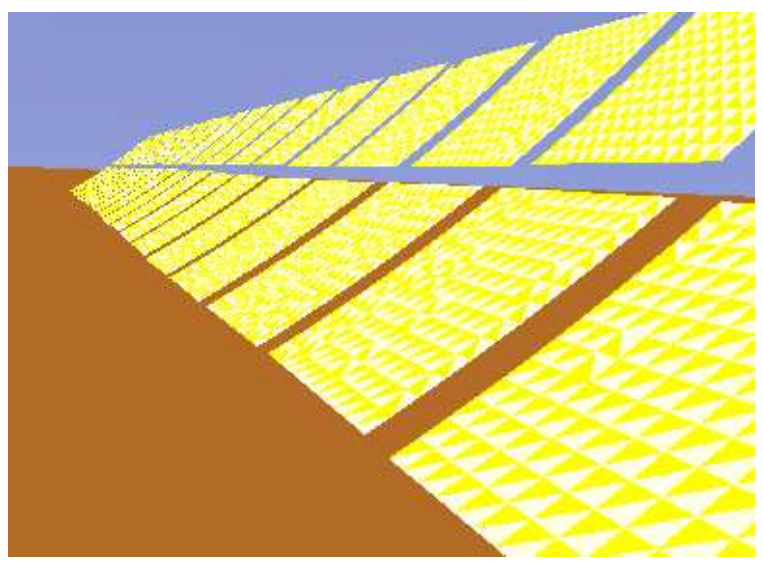

Figura 6: Facetas generadas mediante grupos de triángulos, para el estudio de fotogrametría.

\section{CAMPO DE HELIOSTATOS}

En una planta CSP de torre, el receptor se encuentra localizado en la zona superior de una torre. Desde allí, el fluido caloportador, se bombea hasta el sistema de almacenamiento y el ciclo termodinámico. Alrededor de la torre, se sitúan los espejos, llamados heliostatos, y que enfocan la radiación del Sol hacia el receptor.

Los heliostatos siguen la trayectoria del Sol, gracias a dos grados de libertad (un giro en sentido radial y otro en elevación). Cada uno de los heliostatos está formado por una o varias facetas. 


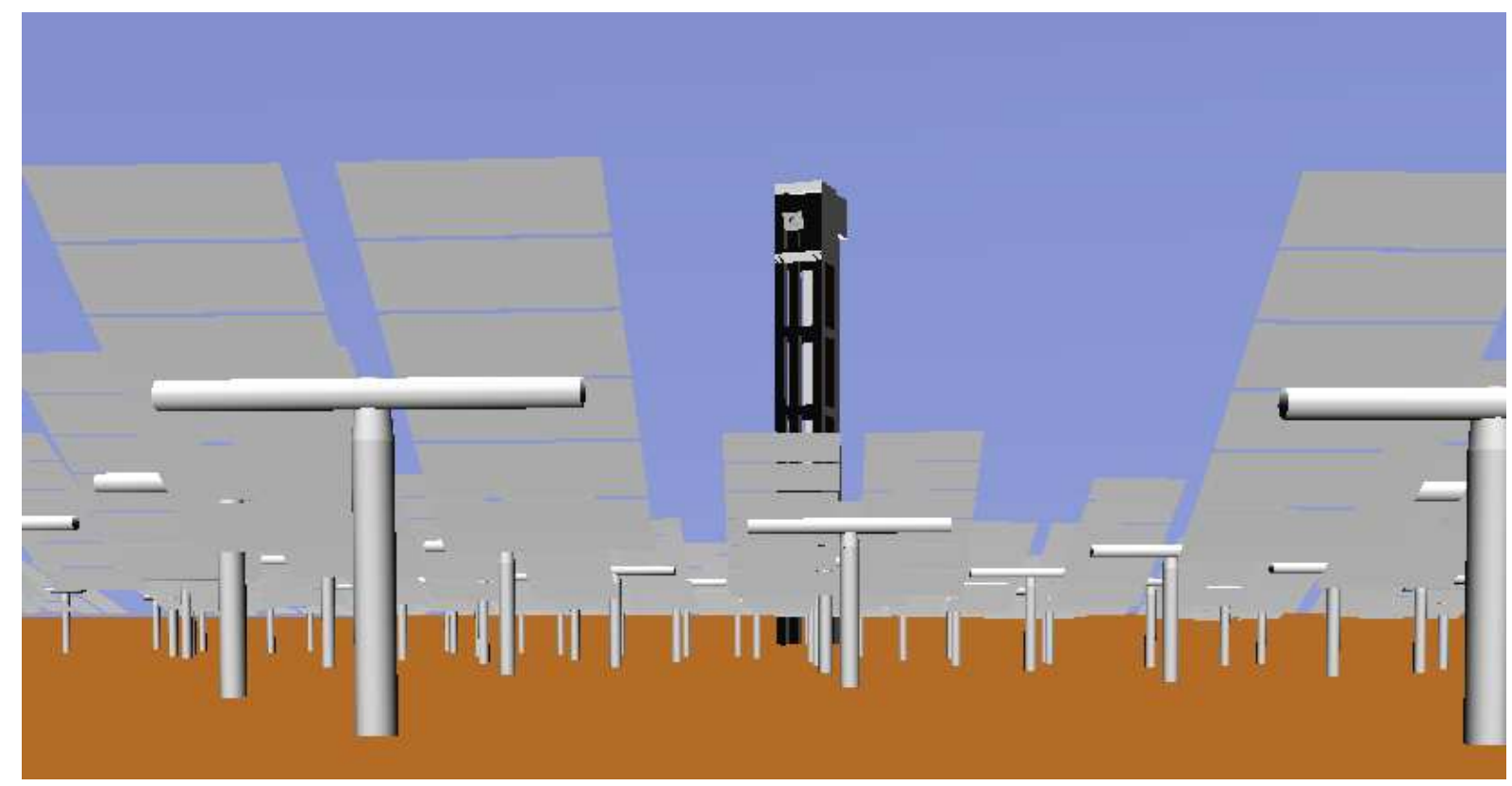

Figura 7: Campo de heliostatos y torre receptora, modelados en Tonatiuh.

El área total de los espejos de un único heliostato, puede superar los $150 \mathrm{~m}^{2}$, aunque la tendencia actual es construir heliostatos pequeños (de menos de $10 \mathrm{~m}^{2}$ ) y monofacetas. El campo de heliostatos puede ser considerablemente extenso (con decenas de miles de heliostatos), y los heliostatos más alejados encontrarse a más de un kilómetro y medio de distancia de la torre. La figura 7 muestra una planta CSP de torre, modelada con Tonatiuh.

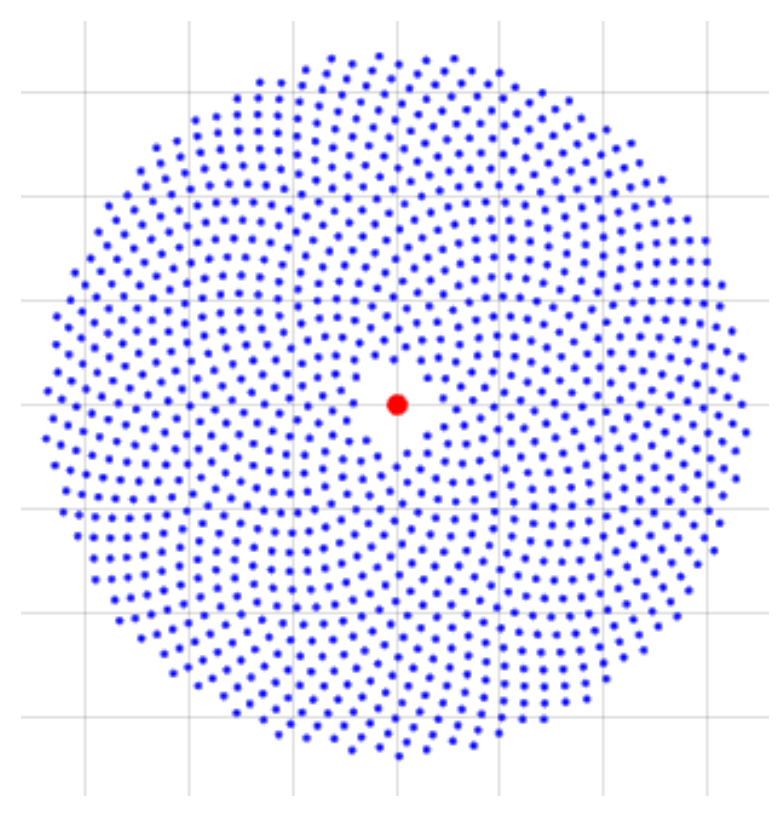

Figura 8: Campo de heliostatos siguiendo una distribución basada en la espiral de Fermat (método biomimético). En rojo se indica la posición de la torre, mientras que en azul se indica la posición de los heliostatos.
La forma en que los heliostatos se distribuyen a lo largo de una planta, es un tema ampliamente estudiado. Básicamente existen dos métodos para el cálculo de la posición de los heliostatos: 1) Métodos del tipo "radial staggered", basados en una distribución tresbolillo. Hoy día son los métodos más populares, y corresponden a distintas variantes del trabajo original propuesto en la década de los 70 [13]. Y 2) Métodos del tipo biomiméticos, basados en crecimientos y patrones naturales. La figura 8 muestra una distribución biomimética, mientras que la figura 11 muestra una distribución "radial staggered".

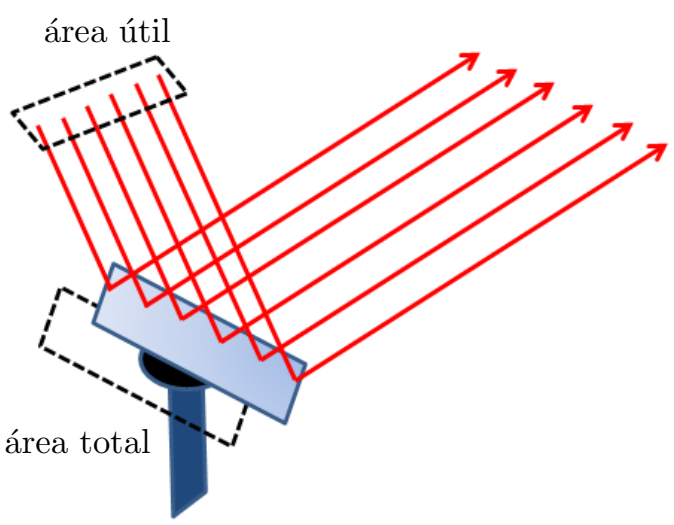

Figura 9: Representación gráfica del efecto coseno.

La eficiencia de un campo de heliostatos varía a lo largo del día, según cambia la posición relativa Sol - heliostato - receptor. Para que los rayos reflejados 
incidan correctamente en el receptor, los heliostatos deben situarse con un cierto ángulo respecto de la perpendicular de los rayos incidentes. Cuanto mayor sea este ángulo, menor es la superficie útil y menor es el flujo total reflejado. Este efecto recibe el nombre de efecto coseno, y se representa gráficamente en la figura 9 .

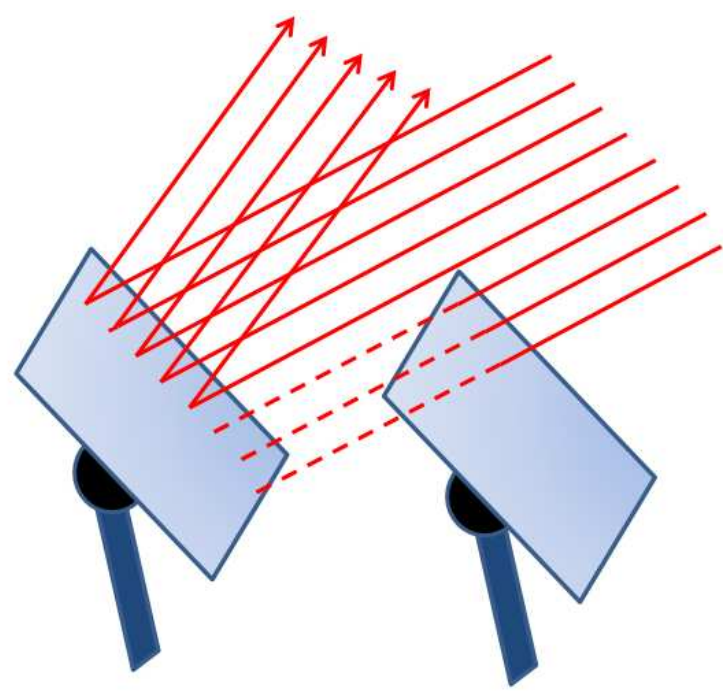

(a) Sombras

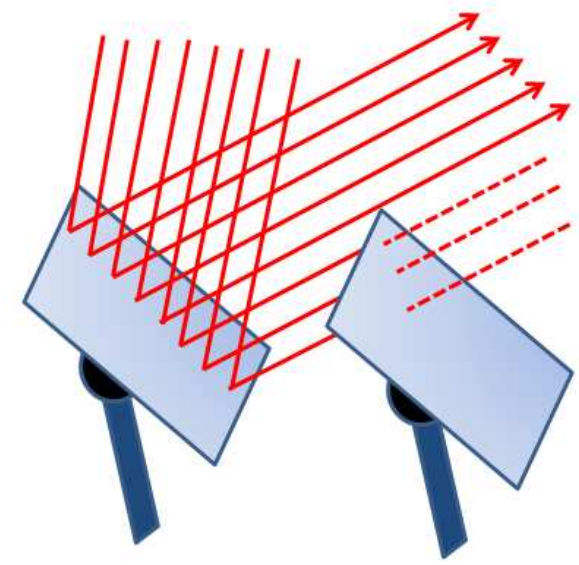

(b) Bloqueos

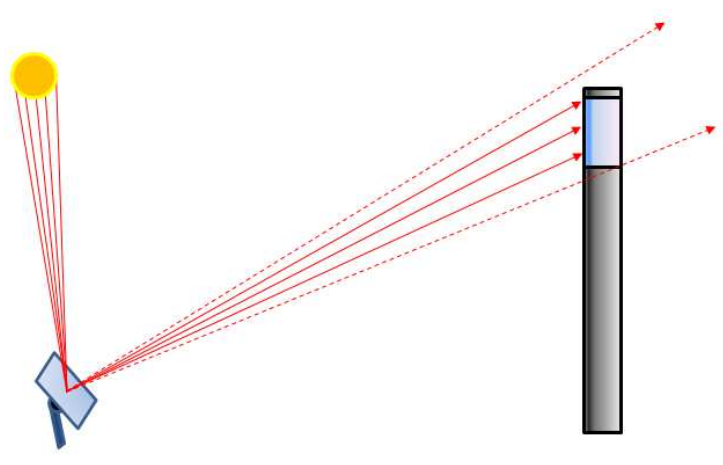

(c) Desbordamiento

Figura 10: Pérdida de eficiencia debido a distintos efectos.
Otros factores que influyen negativamente en la eficiencia de un campo de heliostatos son: 1) Las sombras que se pueden hacer entre sí los heliostatos y otras construcciones del campo (entre ellas, la propia torre). 2) El bloqueo de rayos reflejados, que inciden con otros heliostatos y construcciones. 3) El desbordamiento de rayos, que caen fuera del receptor, especialmente común para los heliostatos más alejados. 4) La atenuación atmosférica. 5) Las deformaciones y errores de apunte de los heliostatos. Causadas, por ejemplo, por el efecto del viento. Etcétera. La figura 10 representa gráficamente algunos de estos efectos.

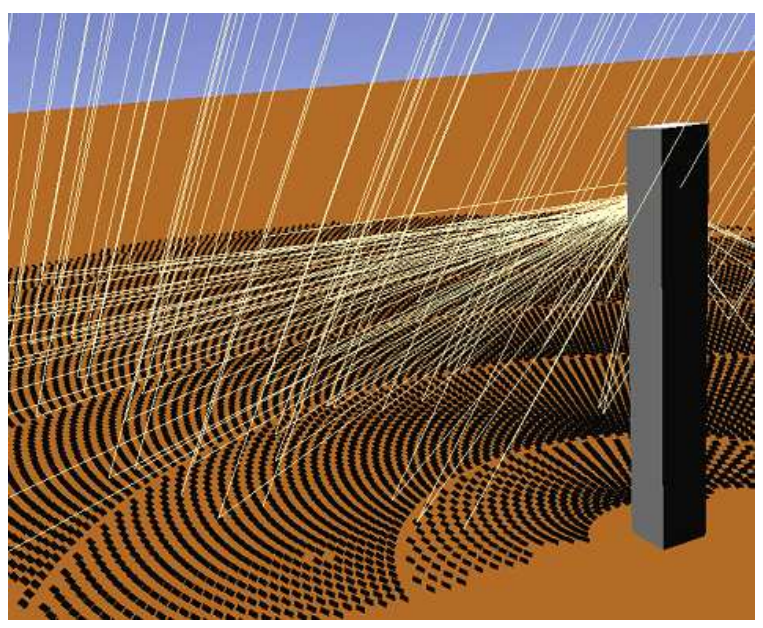

Figura 11: Caminos estocásticos recorridos por los rayos solares, en un campo de heliostatos.

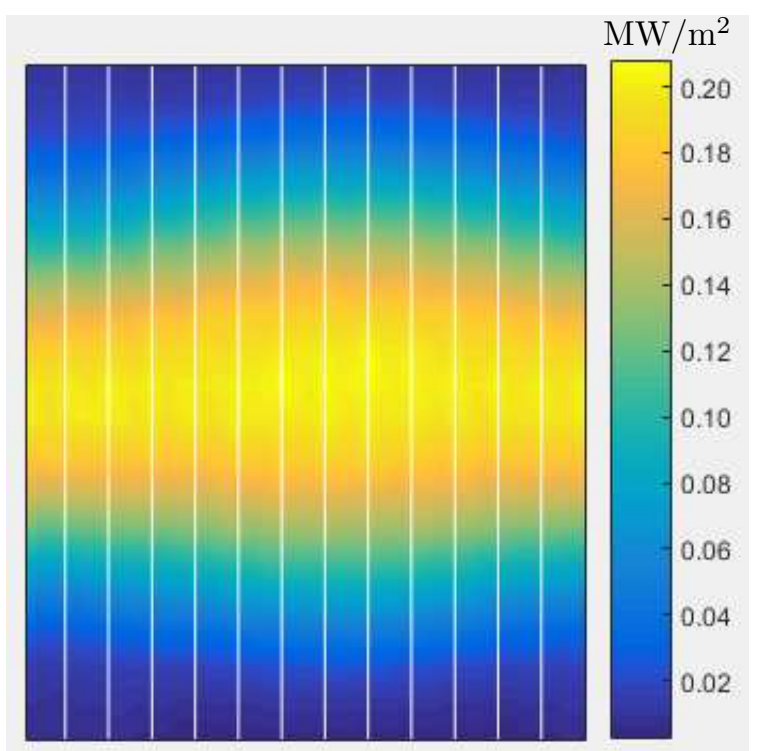

Figura 12: Ejemplo del mapa de flujo de un receptor cilíndrico, calculado con Tonatiuh.

Igual que en el caso anterior, el cálculo de la radiación incidente y que llega al receptor, se puede 
realizar fácilmente mediante simulación de Monte Carlo. La figura 11 muestra el trazado de algunos rayos en un campo de heliostatos, mientras que la figura 12 muestra el cálculo del mapa de flujo de un receptor cilíndrico, situado en el campo de heliostatos representado en la figura 8. La simulación correspondiente se realizó para una posición del Sol (azimut $180^{\circ}$, elevación $70^{\circ}$ ), con todos los heliostatos enfocados en la generatriz central.

Además, Tonatiuh puede ser útil, por ejemplo para: 1) La estimación de la eficiencia óptica de una planta CSP de torre, teniendo en cuenta todas las pérdidas por sombras, bloqueos, efecto coseno, etc. 2) Para el diseño tanto de los heliostatos, como de su localización en el campo, estimando cuáles son las posiciones con mayores pérdidas. Y 3) $\mathrm{Pa}-$ ra el cálculo de estrategias de apunte. Teniendo en cuenta la posición del Sol, las condiciones atmosféricas, las restricciones del receptor (flujo máximo pico, gradientes máximos de temperatura, tanto espaciales como temporales...), etc.

\section{TRABAJO FUTURO}

El proyecto de Tonatiuh está en continua evolución, y actualmente existen varias mejoras que se pretenden llevar a cabo, en un plazo de tiempo corto. En particular, se quiere:

- Extender la validación de Tonatiuh, con nuevos datos reales de plantas CSP (campos de heliostatos, captadores cilindro parabólicos, etc.). Y realizar un estudio estadístico de los resultados, de manera extensiva y somera.

- Reducir de manera considerable el tiempo de cálculo. Principalmente, utilizando técnicas de simulación en GPU [17]. Actualmente, el código de Tonatiuh está optimizado para simulación en CPU. Pero la naturaleza del problema, hace que sea especialmente indicada su programación en GPU, cuyo microprocesador puede tener cientos de núcleos diseñados para operar en paralelo.

- Incluir nuevos modelos en el proyecto Tonatiuh. En especial, ampliar la lista de modelos del Sol.

\section{CONCLUSIONES}

Este documento ha presentado, a través de dos ejemplos, las capacidades del software Tonatiuh, un trazador de rayos basado en simulación de Monte Carlo. Está especialmente indicado para el cálculo de radiaciones incidentes en plantas termosolares, y tiene en cuenta los defectos de superficies, las propiedades ópticas de los materiales, la atenuación atmosférica, las sombras, los bloqueos, el desbordamiento, y un largo etcétera. Tonatiuh trabaja bajo licencia GNU GPL, es de código abierto y gratuito. Desde el CENER se fomenta su uso y desarrollo, especialmente a los centros tecnológicos, universidades y empresas del sector.

\section{Referencias}

[1] Ballestrin, J., Marzo, A., (2012) "Solar radiation attenuation in solar tower plants", Solar Energy, (86) pp 388-392.

[2] Biggs, F., Vittitoe, C.N., "Helios model for the optical behavior of reflecting solar concentrators", Sandia labs, Albuquerque, NM, USA.

[3] Birol, F., (2008) "World energy outlook", International Energy Agency.

[4] Blanco, M., Mutuberria, A., Garcia, P., Gastesi, R., Martin, V., (2009) "Preliminary validation of Tonatiuh", SolarPACES, Berlin, Germany.

[5] Blanco, M., Mutuberria, A., Martinez, D., (2010) "Experimental validation of Tonatiuh using the Plataforma Solar de Almeria secondary concentrator test campaing data", SolarPACES, Perpignan, France.

[6] Bode, S.J., Gauche, P., (2012) "Review of optical software for use in concentrating solar power systems", South African Solar Energy Conference, Stellenbosch, South Africa.

[7] Buie, D., Dey, C.J., Bosi. S., (2003) "The effective size of the solar cone for solar concentrating systems", Solar Energy, (74) pp 417-427.

[8] Dellin, T.A., Fish, M.J., (1979) "DELSOL A code for central receiver performance and optimization calculations", Sun, (1) pp 12331237.

[9] Garcia, P., Ferriere, A., Bezian, J.J., (2008) "Codes for solar flux calculations dedicated to central receiver system applications: A comparative review", Solar Energy, (82) pp 189197.

[10] Garg, H. P., Mullick, S. C., Bhargava, V.K., (2012) "Solar thermal energy storage", Springer Science \& Business Media.

[11] Ho, C.K., Mahoney, A.R., Ambrosini, A., Bencomo, M., Hall, A., Lambert, T.N., (2014) "Characterization of Pyromark 2500 paint for high-temperature solar receivers", 
Journal of Solar Energy Engineering, (136) pp 014502.

[12] Kalogirou, S.A., (2013) "Solar energy engineering: processes and systems", Academic Press.

[13] Lipps, F.W., Vant-Hull, L.L., (1978) "A cellwise method for the optimization of large central receiver systems", Solar Energy, 20(6) pp 505-516.

[14] Lovegrove, K., Stein, W., (2012) "Concentrating solar power technology: principles, developments and applications", Elsevier.

[15] Mutuberria, A., Monreal, A., Albert, A., Blanco. M., (2011) "Results of the empirical validation of Tonatiuh at Mini-Pegase CNRSPROMES facility", SolarPACES, Granada, Spain.

[16] Mutuberria, A., Monreal, A., Blanco, M., Sanchez, M., Ferriere, A., (2012) "Modelling and structure deformation analysis of a heliostat at Mini-Pegase CNRS-PROMES facility", SolarPACES, Marrakech, Morocco.

[17] Garcia M.P., Zamarreño J.M., (2012) "Optimización multiarranque en paralelo sobre GPU", XXXIII Jornadas de Automática, Vigo, Spain.

[18] Peterseim, J.H., White, S., Tadros, A., Hellwig, U., (2013) "Concentrated solar power hybrid plants, which technologies are best suited for hybridisation?", Renewable Energy, (57) pp 520-532.
[19] REN21, (2015) "Renewables 2015 global status report", Paris: REN21 Secretariat.

[20] Roccia, J.P., Piaud, B., Coustet, C., Caliot, C., Guillot, E., Flamant, G., Delatorre, J., (2012) "SOLFAST, a Ray-Tracing MonteCarlo software for solar concentrating facilities", Journal of Physics, (369) pp 012029.

[21] Sengupta, M., Wagner, M. (2012) "Atmospheric attenuation in central receiver systems from DNI measurements", ASES World Renewable Energy Forum, Denver, CO, USA.

[22] Urrizburu, A., (2016) "Optimisation and computational study of a solar thermal power plant collector using air as working fluid", Universidad Pública de Navarra.

[23] Vant-Hull, L.L., Pitman, C.L., (1988) "Central receiver system optimization under an allowable flux constraint", International Symposium on Solar Thermal Technology Research, Santa Fe, NM, USA.

[24] Wendelin, T., (2003) "SolTRACE: a new optical modeling tool for concentrating solar optics", ASME 2003 International Soalr Energy Conference, Kohala Coast, HI, USA.

[25] Yellowhair, J., Ortega, J.M., Christian, J.M., Ho, C.K., (2014) "Solar optical codes evaluation for modeling and analyzing complex solar receiver geometries", SPIE Optical Engineering + Applications, San Diego, CA, USA. 